\title{
Kaonic nuclear state search at J-PARC
}

\author{
Masahiko Iwasaki ${ }^{1, \star}$ \\ ${ }^{1}$ RIKEN, Wako-shi, Saitama 351-0198, Japan
}

\begin{abstract}
At J-PARC, there are two experimental programs, E15 and E27, to search for the simplest kaonic bound nuclear state in $\bar{K} N N$ system. These two experiments were proposed based preceding experiments reporting candidates of such state, although the experimental results are not quite consistent to each other. These two experimental programs utilizing different reaction channels, ${ }^{3} \mathrm{He}\left(K^{-}, n\right)$ reaction by $\mathrm{K}^{-}$momentum at $1 \mathrm{GeV} / c$ for E15, and $d\left(\pi^{+}, K^{+}\right)$reaction by $\pi^{+}$momentum at $1.7 \mathrm{GeV} / c$ for $\mathrm{E} 27$, to resolve this puzzling situation. In this paper, we overview these two experimental results as flat as possible.
\end{abstract}

\section{Introduction}

The $\bar{K} N$ interaction is known to be strongly attractive from low-energy scattering data [1] and X-ray spectroscopy of kaonic atoms [2]. On the other hand, there exist a well known pole, $\Lambda(1405)$, slightly below the $\bar{K} N$ mass threshold. This $\Lambda(1405)$ resonance is known that it is very difficult to be treated as a simple excited state of $\Lambda$ hyperon, and it is widely accepted that the $\Lambda(1405)$ is $K^{-} p$ bound state / penta-quark like structure or couple to these states. If that is true, the natural extension is that the kaon will form a nuclear bound state, helped by the existence of the $\Lambda(1405)$. Accordingly, such states are predicted and a high density matter formation, exceeding the normal nuclear density, is expected in such states [3]. Therefore, observation of a kaonic nuclear bound state would provide definitive information on the nature of $\Lambda(1405)$, and nuclear physics at very high density.

Both theoretical and experimental studies have been made in the last decade for the simplest kaonic nuclear state $\bar{K} N N$. However, the results do not seem to be consistent each other. Theoretically, all calculations predict the existence of a bound state, but the predicted $\bar{K} N N$ pole positions are scattered depending on $\bar{K} N$ interaction models. For the energy-independent model, the binding energy is reaching up to $50 \sim 100 \mathrm{MeV}$, while in energy-dependent case, it becomes weaker to be $10 \sim 30 \mathrm{MeV}$. The widths are also widely scattered over $30 \sim 110 \mathrm{MeV}$. Experimentally, there are few positive reports on peak structure observations as for the candidate at $\sim 100 \mathrm{MeV}$ below $\bar{K} N N$ threshold. The first report from FINUDA group showing a peak structure in the back-to-back $\Lambda p$ invariant mass spectra via the stopped kaon reaction on ${ }^{6} \mathrm{Li},{ }^{7} \mathrm{Li}$, and ${ }^{12} \mathrm{C}$ targets [4], having binding energy (B.E.) $\sim 115 \mathrm{MeV}$, and a width $(\Gamma) \sim 70 \mathrm{MeV}$. The DISTO group conducted a $p p$ collision experiment. observed $\bar{K} N N$ candidate at B.E. $\sim 100 \mathrm{MeV}$, having $\Gamma \sim 120 \mathrm{MeV} / c^{2}$ [5]. Conversely, no significant structure was observed in a SPring-8/LEPS $\gamma$ induced inclusive experiment [6] or in a proton-proton interaction by the HADES/GSI collaboration [7].

^e-mail: masa@riken.jp 
In these experimental spectra, the most significant structure was found in DISTO. They examined $<K^{-} p p>$ formation using $p p \rightarrow K^{+}<K^{-} p p>$ reaction at $T_{p}=2.85 \mathrm{GeV}$. The momentum transfers $q_{K}$ to $\left\langle K^{-} p p>\right.$ system, viewed from $p p$ rest-frame (CM-frame), are as low as $\sim 300$ and $\sim 430 \mathrm{MeV} / c$ at B.E. $\sim 0$ and $\sim 100 \mathrm{MeV}$, respectively. The low $q_{K}$ will enhance the $K^{-}$sticking probability to $p p$ system, helped by the $\Lambda(1405)$ pole just below the $K^{-} p$ mass threshold. If the decay branch, $<K^{-} p p>\rightarrow \Lambda p$, is relatively large, then the signal observation through $K^{+} \Lambda p$ final state, by the $\Lambda p$ invariant mass (or $K^{+}$missing mass).

There are, however, many concerns in DISTO result. Their $p p$ collision energy is just above the production threshold of $N^{*}$, which decay strongly to $K^{+} \Lambda$ (such as $N^{*}(1710)$ ). In the reaction near the formation threshold, such $N^{*}$ s are nearly at-rest in the $C M$-frame, so it is rather natural to have kinematical structure in the invariant mass spectrum of $\Lambda p$ (or the missing mass of $K^{+}$), in the chain reaction of $p p \rightarrow N^{*+} p \rightarrow K^{+} \Lambda p$, even without $K^{-} p p$ resonance of $p p \rightarrow K^{+}<K^{-} p p>\rightarrow K^{+} \Lambda p$. The $\Lambda p$ invariant mass peak stand just on the $\Sigma \pi p$ mass threshold having no threshold effect, which indicates that the state do not couple to the $\Sigma \pi p$ channel. This is rather strange, because $\left\langle K^{-} p p>\right.$ should strongly couples with $\Lambda(1405)-p$, and the major decay channel of $\Lambda(1405)$ is $\Sigma \pi$. The spectrum is also insensitive to the $K^{-} p p$ mass threshold, while it is more natural to have non-resonant process above the threshold (quasi-free $K^{-} p p$ formation), because the reaction chain of $p p \rightarrow K^{+} K^{-} p p$ (or $\left.K^{+} Y^{(*)} p\right) \rightarrow K^{+} \Lambda p$ could happen at relatively low momenta of those particles in CM-frame. And finally, in contrast to the DISTO result, no peak structure found in same $p p$ collision at different kinetic energy $T_{p}=3.5 \mathrm{GeV}$ by HADES. The $q_{K}$ is $\sim 550$ and $\sim 650 \mathrm{MeV} / c$ viewed from CM-frame at B.E. $\sim 0$ and $\sim 100 \mathrm{MeV}$, respectively. The transfer of $\sim 650 \mathrm{MeV} / c$ is rather large to form a bound state efficiently, but it would be still sizable if the B.E. of $\left\langle K^{-} p p\right\rangle$ is as large as $\sim 100 \mathrm{MeV}$ in comparison with that of $\sim 430 \mathrm{MeV} / c$ at DISTO.

\section{Kaonic nuclear state search at J-PARC}

To clarify the situation, two experimental groups, E15 and E27, conducted experiments at J-PARC searching for $\bar{K} N N$ bound state. J-PARC E15 and E27 utilizing different reaction channels. The E15 is utilizing ${ }^{3} \mathrm{He}\left(K^{-}, n\right) X$ reaction at $K^{-}$momentum of $1 \mathrm{GeV} / c$, while the E27 is utilizing $d\left(\pi^{+}, K^{+}\right) X$ reaction at $\pi^{+}$momentum of $1.69 \mathrm{GeV} / c$. E27 is conducted in 2012 earlier than E15, because high intensity $\pi^{+}$beam of $1.69 \mathrm{GeV} / c$ can be easily obtained at K1.8 beam line at J-PARC. E15 was assigned as Day-One-Experiment at J-PARC, but the first run $\left(\mathrm{E} 15^{1 s t}\right.$ ) was executed only in 2013, since it requires high intensity kaon beam.

\subsection{J-PARC E27}

The E27 was conducted to examine the striking result of DISTO, that the $\left\langle K^{-} p p>\right.$ could be bound as astonishingly strong as $\sim 100 \mathrm{MeV}$, having large cross section, and having a relatively large decay branch to $\Lambda p$ [8]. The $\bar{s} s$-pair is produced at the reaction as it is like in $p p$ collision, but the kinematics is much different. In the $\pi^{+} d \rightarrow K^{+} K^{-} p p$ (or $K^{+} X$ ) reaction, the two proton in $X=<K^{-} p p>$ come from the deuteron target, so the $q_{K}$ to the $\left\langle K^{-} p p>\right.$ system should be calculated in the deuteron restframe (Lab.-frame). The difference of the kinematics makes energy dependence to the $q_{K}$ opposite, namely $730 \mathrm{MeV} / c$ for B.E. $=0$, and $570 \mathrm{MeV} / c$ for B.E. $=100 \mathrm{MeV}$. Thus, E27 is kinematically more like HADES rather than DISTO.

In E27, forward $K^{+}$from $d\left(\pi^{+}, K^{+}\right) X$ reaction is identified and momentum analyzed by SKS, a large acceptance $(\sim 100 \mathrm{msr})$ superconducting spectrometer covering from 0.8 to $1.3 \mathrm{GeV} / \mathrm{c}$ in $K^{+}$ momentum, and from 2 to 16 degree in angle in the Lab.-frame. To detect decay protons from $X$ for $p_{p}>250 \mathrm{MeV} / c$, E27 equipped 6 sets of range counter arrays (RCA) as shown in figure 1. 


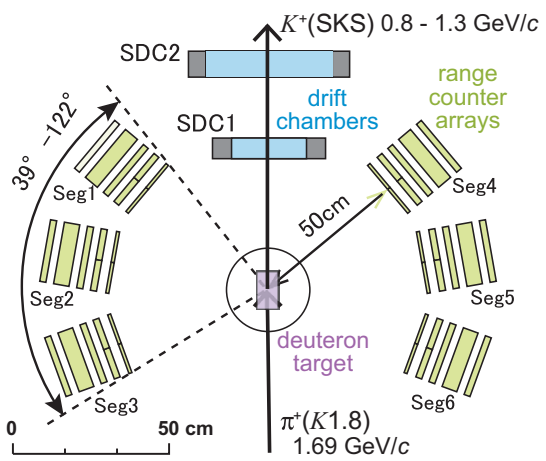

Figure 1. The proton RCA is made of five layers of plastic scintillation counters of about $12 \mathrm{~g} / \mathrm{cm}^{2}$ in total $(1+2+2+5+2 \mathrm{~cm}$ in thickness). Three sets of the range counters are placed in the horizontal plane both left and right side, viewed from incident $\pi^{+}$beam.

In figure 2 , the $K^{+}$missing mass spectra are shown. Fig. 2 (left) is the spectrum for inclusive $d\left(\pi^{+}, K^{+}\right) X$ reaction. As it is naturally expected, $d\left(\pi^{+}, K^{+}\right) X$ reaction is sensitive to the $K^{+} Y^{(*)} p$ formation. In fact, quasi-free hyperon formations are clearly seen as $K^{+} \Lambda p$ and $K^{+} \Sigma N$ final states below $\sim 2.2 \mathrm{GeV} / c^{2}$. It also shows that the yields associated with additional pions, channel open at $\sim 2.2$ and $\sim 2.27 \mathrm{GeV} / c^{2}$, are relatively week, when one require the forward $K^{+}$formation as it is the case in E27. Fig. 2 (right) is that for one proton-tagged event in RCA. In the spectrum, $K^{+} \Lambda p$ channel is suppressed as shown in the figure, while $K^{+} \Sigma N$ channel still remains especially near the $\Sigma N$ threshold. The rest of $K^{+} \Sigma N$ channel is also tagged more efficiently than $K^{+} \Lambda N$, helped by the energy transfer to the spectator nucleon, in the successive $\Sigma N \rightarrow \Lambda N$ conversion.
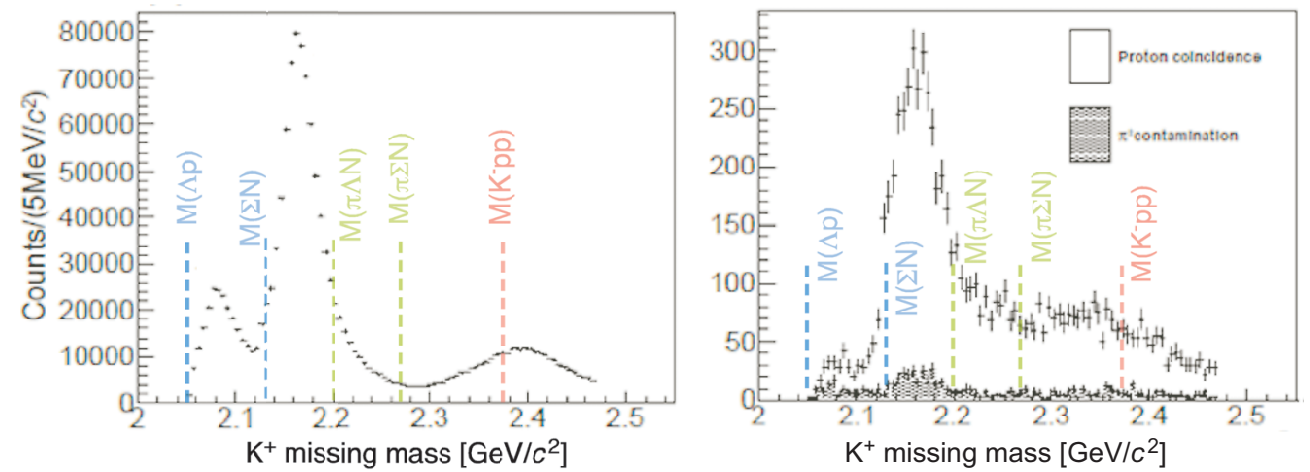

Figure 2. Left) A $K^{+}$missing mass $M(X)$ spectrum for the inclusive $d\left(\pi^{+}, K^{+}\right) X$ reaction. Threshold energy for several related channels, where each channel open energetically, are plotted in dashed lines. Right) A subset of the same inclusive spectrum, in which at least one proton hits in one of RCA.

The other difference is the spectral strength in the energy region from $M(\pi \Lambda p)$ to $M\left(K^{-} p p\right)$. To understand the difference, missing mass analysis was applied for $d\left(\pi^{+}, K^{+} p p\right) Y$ to the two proton identified events on RCA. If $K^{+} \Lambda p$ is the final state of $\pi^{+} d$ reaction, then the missing mass analysis of the $d\left(\pi^{+}, K^{+} p p\right) Y$ kinematics should yield that $Y=\pi$. If the final state is $K^{+} \Sigma^{0} p$, then the analysis gives that $Y=\pi \gamma$. Thus, one can specify the final state of the reaction using kinematics, although the statistics of the $p p$-coincidence events is drastically reduced due to the limited RCA solid angle. One should also be careful to the kinematical deformation due to the RCA coverage both in angle and energy threshold. 

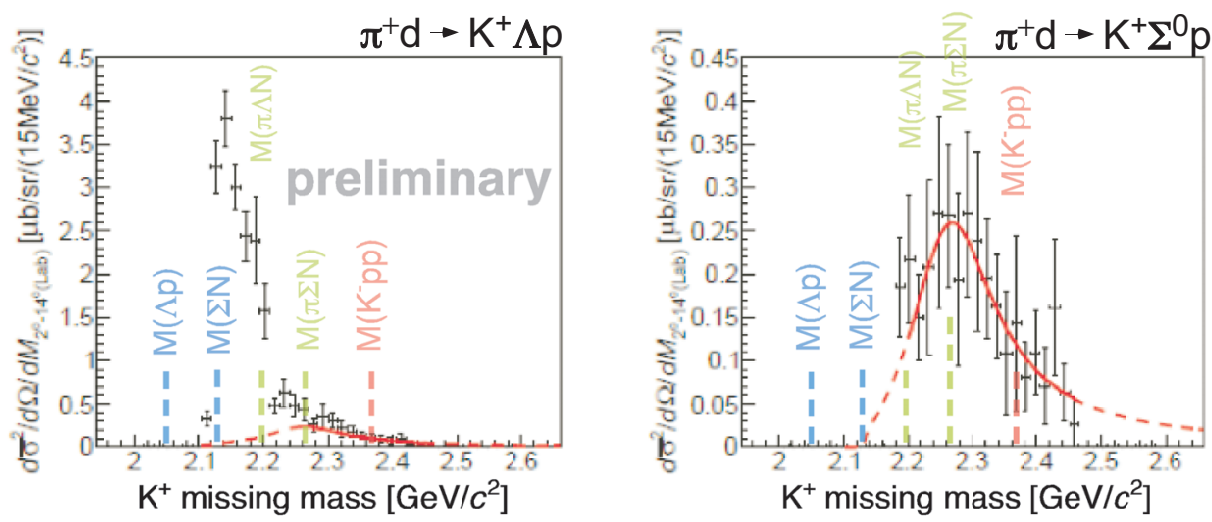

Figure 3. The $K^{+}$missing mass spectrum of $d\left(\pi^{+}, K^{+}\right) X$ reaction for $K^{+} \Lambda p$ final state (left), and that for $K^{+} \Sigma^{0} p$ final state (right),

Fig. 3 shows the $K^{+}$missing mass spectra for $K^{+} \Lambda p$ final state (left) and that for $K^{+} \Sigma^{0} p$ final state (right). In Fig. 3 (left), there is a structure from $M(\Sigma N)$ up to $M(\pi \Lambda N)$, which was interpreted that the structure is formed due to the $\Sigma N$-cusp and $\Sigma N \rightarrow \Lambda N$ conversion. In Fig. 3 (right), there is a wide event distribution above $M(\pi \Lambda N)$, which extended smoothly even beyond the $M\left(K^{-} p p\right)$ threshold. This broad structure in both spectra were fitted by a Breit-Wigner formula in the energy region indicated by the solid curve. The result of the peak position and the width are roughly consistent with DISTO, which gives extremely deep binding energy of about $100 \mathrm{MeV}$. On the other hand, all the concerns to the DISTO result would remain as they were. There would be another concern, why $\pi^{+} d$ reaction is sensitive to $\Sigma N$-cusp and $\Sigma N \rightarrow \Lambda N$ conversion, while it is not in $p p$ collision.

\subsection{J-PARC E15}

As for the other attempt to for $\left\langle K^{-} p p>\right.$ much more definitively, E15 experiment on the $K^{-}+{ }^{3} \mathrm{He}$ reaction is underway at J-PARC in a stepwise manner. The experimental approach of E15 is much different from other experimental researches. Firstly, the strangeness is introduced as a $K^{-}$beam at $1 \mathrm{GeV} / c$, where the $\bar{K} N$ elastic cross sections are quite large $\left(e . g . n\left(K^{-}, n\right) K^{-} \sim 5 \mathrm{mb} @ 1 \mathrm{GeV} / c\right)$. Therefore, elastic ${ }^{3} \mathrm{He}\left(K^{-}, n\right) X$ reaction channel, a neutron knockout reaction, is dominant. In this reaction, the $q_{K}$ to $\left\langle K^{-} p p>\right.$ system, viewed from spectator protons' rest-frame (Lab.-frame) in $K^{-3} \mathrm{He} \rightarrow n+K^{-} p p$, is as small as $230 \mathrm{MeV} / c$ for B.E. $=0$, and it is still $330 \mathrm{MeV} / c$ even for B.E. $=100 \mathrm{MeV}$. The $q_{K}$ is smaller by about $100 \mathrm{MeV} / c$ than those in DISTO. Thus, one can expect even more efficient formation of the $\left\langle K^{-} p p\right\rangle$ system in E15 than DISTO. The experimental setup is consist from three components; forward neutron counter array (NC), forward proton spectrometer using beam sweeping magnet, and cylindrical detector system (CDS) surrounding the liquid ${ }^{3} \mathrm{He}$ target. In the E15 kinematics, $\left\langle K^{-} p p>\right.$ system is formed almost at-rest (as it is described, $230 \mathrm{MeV} / c$ backward at B.E. $=0$ ), so the decay products can be efficiently detected by the CDS [9]. Another remarkable point in this reaction is that it is naturally insensitive to the production and the decay of $N^{*} \rightarrow K^{+} \Lambda$, because of the $Q$-value.

The first paper was published on the semi-inclusive neutron spectrum ${ }^{3} \mathrm{He}\left(K^{-}, n\right) X$ in the forward direction [10]. The missing mass spectrum of the neutron is shown in Fig. 4. As it is expected, kaon 


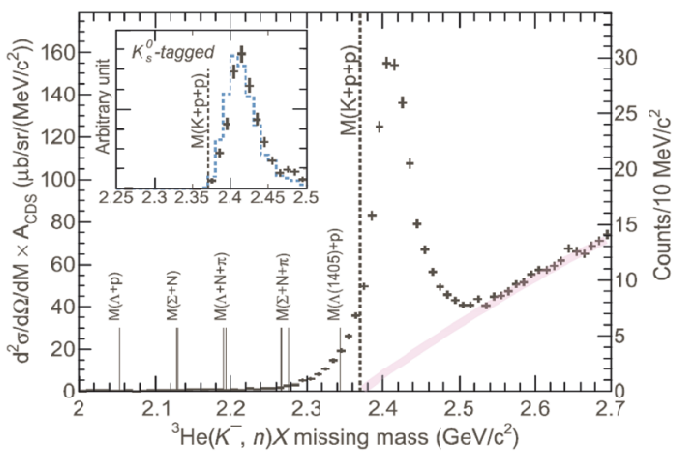

Figure 4. The semi-inclusive neutron spectrum of the ${ }^{3} \mathrm{He}\left(K^{-}, n\right) X$ reaction in the forward direction. To identify the kaon reaction point, one charged particle track is requested in CDS. In the semi-inclusive spectrum, a long tail is observed below $M(K p p)$ threshold. To show pure kaon quasi-elastic scattering process, $K^{-}$" $p$ " $\rightarrow \bar{K}^{0} n$, out from the inclusive reactions, $K_{s}^{0}$ tagged spectrum is shown as inset. The quasi-elastic channel start right on the $M(K p p)$ threshold, having a width caused by the Fermi-motion of the incident proton in the nucleus.

quasi-elastic channel is clearly seen at around $2.4 \mathrm{GeV} / c^{2}$. In spite of the lower $q_{K}$ to $<K^{-} p p>$ system, no significant peak structure is observed around $M(\pi \Sigma p)$ energy region, as it is the case in DISTO or E27. Instead, there is a long tail below $M(K p p)$ threshold, extended to $M(\pi \Sigma p)$ beyond $M(\Lambda(1405) p)$ threshold. This tail cannot be explained by a simple extrapolation from the high excitation continuum (large missing mass region), as shown in the figure. This indicate that there is a strong attractive interaction between $\bar{K} N$, since such a long tail $(\sim 100 \mathrm{MeV})$ is very difficult to be formed simply by the imaginary part of the $\bar{K} N$ interaction. In contrast to E27 spectrum given in Fig. 2 (left), no strong yield is observed near the $M(\Lambda p)$ nor $M(\Sigma N)$, which indicating that the kaon two-nucleon-absorption process (2NA) is rather weak at $p_{K}=1 \mathrm{GeV} / c$.

The second paper from E15 is focused on the $\Lambda p n$ final state [11]. Since $\left\langle K^{-} p p>\right.$ system is produced almost at-rest, the decay process to $\Lambda p$ can be studied only by CDS information without using $\mathrm{NC}$ for forward neutron. By $\Lambda p$ 4-momenta, one can reconstruct reaction if the event can be kinematically identified as ${ }^{3} \mathrm{He}\left(K^{-}, \Lambda p\right) n$ by the missing mass. Fig. 5 (left) shows the $\Lambda p$ invariant mass
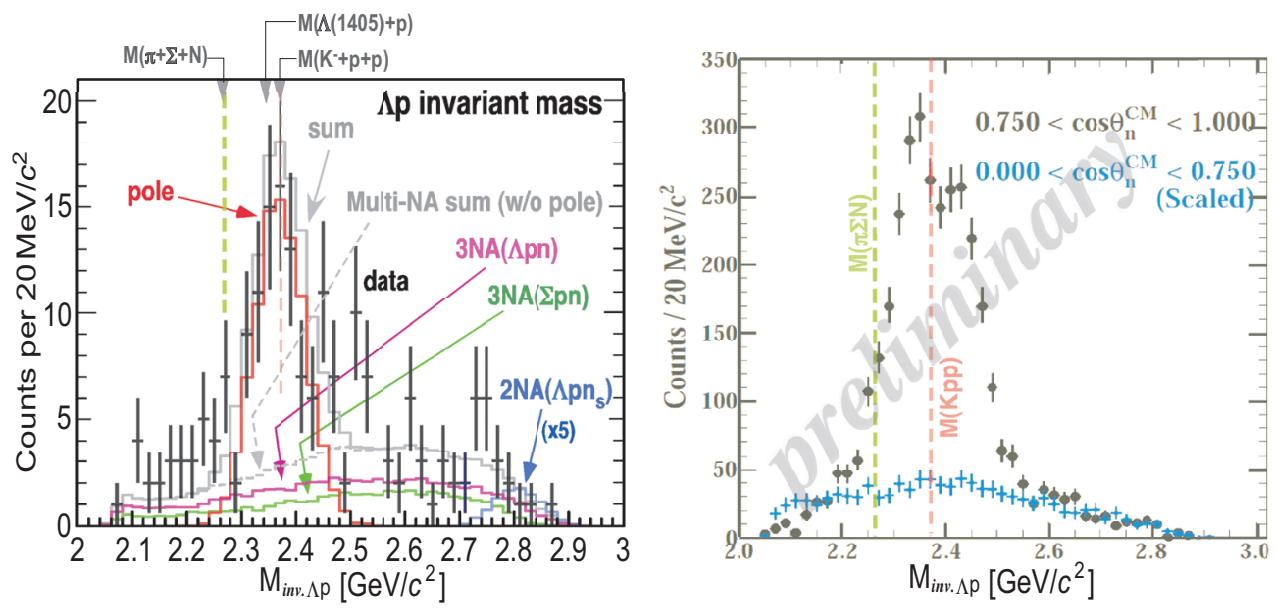

Figure 5. Left) The $\Lambda p$ invariant mass spectrum for ${ }^{3} \mathrm{He}\left(K^{-}, \Lambda p\right) n$ events of the $\mathrm{E} 15^{1 \text { st. }}$ data without selecting neutron emission angle. The spectrum was fitted with single pole together with the multi-nucleon absorption processes represented by \#n-body phase space. Right) Same spectra with 30 times more data using E15 $5^{2 n d}$. data. The peak structure is observed in forward neutron emission events $\left(\cos \theta_{n}^{C M}>0.75\right)$, while very smooth spectrum is observed in large-angle neutron emission events $\left(0<\cos \theta_{n}^{C M}<0.75\right.$ region scaled by $\left.\sim 1 / 2\right)$. 
spectrum for ${ }^{3} \mathrm{He}\left(K^{-}, \Lambda p\right) n$ events of the E15 $5^{1 s t}$ data. Two spectra, given in Fig. 4 and Fig. 5 (left), are observing essentially same quasi-elastic kaon scattering reaction, except for the two differences. One is the final state selection, another is the emission angle of the neutron. Fig. 4 is semi-inclusive by limiting neutron emission angle to be zero by NC, while Fig. 5 is selective to $\Lambda p n$ final state without limiting emission angle, however, the spectral difference is quite clear. Although the statistics in Fig. 5 (left) is quite limited, it is clear that the structure near the $M(K p p)$ threshold observed in Fig. 5 (left) cannot be explained by the simple quasi-elastic kaon scattering like in Fig. 4. Thus, a simplest pole existence is assumed in [11], together with the Gaussian form factor of $\left|\exp \left(-q_{K}^{2} / 2 Q_{K}^{2}\right)\right|^{2}$, where $q_{K}$ is the $q_{K}$ to $<K^{-} p p>$ system and $Q_{K}$ is the fitting parameter. The fit result was $\sim 15 \mathrm{MeV}$ for B.E, width of $\sim 100 \mathrm{MeV}$, and $Q_{K} \sim 400 \mathrm{MeV} / c$.

To understand the observed peak structure in more detail, E15 $5^{2 n d}$ run was conducted in 2015, succeeding in accumulating $\sim 30$ time more data on $\Lambda p n$ final state, as shown in Fig. 5 (right). In the high statistics spectra, internal structure near the $M(K p p)$ become very clear, both below and above the $M(K p p)$. In the E15 ${ }^{1 s t}$ run, the contribution of quasi-elastic kaon reaction above the $M(K p p)$ is assumed to be small because of the final state. However, in comparison with Fig. 4, it is clear that the non-resonant nuclear absorption can happen even above the $M(K p p)$ threshold in the high statistics data of $\mathrm{E} 15^{2 n d}$. The peak structure sandwiched in between $M(\pi \Sigma N)$ and $M(K p p)$ (kaon bound region) is well understood if there exist a kaonic nuclear bound state. The sudden drop off of yield at $M(\pi \Sigma N)$ is consistent that the $<K^{-} p p>$ couples strongly to $\Lambda(1405)-p$, and decay to $\pi \Sigma N$ channel. In this region, free $\Lambda(1405)$ could not contribute by definition. The knockout nucleon exceeds $M(K p p)$ threshold energy, only when $\Lambda(1405)$ forms a bound state with the other spectator proton nearby, and decay to a $\Lambda p$ pair. Fig. 5 (right) also showing that two structures near the $M(K p p)$ threshold are formed only associated with forward neutron emission $\cos \theta_{n}^{C M}>0.75$, where $q_{K}$ is small $\left(Q_{K} \sim 400 \mathrm{MeV} / c\right)$. This is consistent that present peak structures are observed only in E15 experiment, in which lowest $q_{K}$ of $\sim 230 \mathrm{MeV} / c$ is achieved.

E15 group is analyzing the data in detail, to check if the above interpretation has any further concern. In that analysis, the pole position, width and the $q_{K}$ dependence, must be revisited again, because the simple single-pole assumption in [11] is not valid any more.

\section{Acknowledgement}

The author is grateful to J-PARC E15 group, E27 group and all the J-PARC/KEK staff, who contributed those experimental programs.

\section{References}

[1] A. D. Martin, Nucl. Phys. B 179, 33 (1981)

[2] M. Bazzi et al., Phys. Lett. B 704, 113 (2011)

[3] Y. Akaishi and T. Yamazaki, Phys. Rev. C 65, 044005 (2002)

[4] M. Agnello et al., Phys. Rev. Lett. 94, 212303 (2005)

[5] T. Yamazaki et al., Phys. Rev. Lett. 104, 132502 (2010)

[6] A. O. Tokiyasu et al., Phys.Lett. B 728, 616 (2014)

[7] L. Fabbietti et al., Nucl. Phys. A 914, 60 (2013)

[8] Y. Ichikawa et al., Prog. Theor. Exp. Phys. 021D01 (2015)

[9] K. Agari et al., Prog. Theor. Exp. Phys. 02B011 (2012)

[10] T. Hashimoto et al., Prog. Theor. Exp. Phys. $061 D 01$ (2015)

[11] Y. Sada et al., Prog. Theor. Exp. Phys. 051D01 (2016) 